\title{
Sex differences in chiasma frequency and distribution in natural populations of Eyprepocnemis plorans containing $B$-chromosomes
}

\author{
M. I. Cano, \\ G. H. Jones $\dagger$ and \\ J. L. Santos*
}

\author{
* Departamento de Genetica, Faculdad de Biologia, \\ Universidad Complutense, 28040 Madrid, Spain. \\ $\dagger$ Department of Genetics, University of Birmingham, \\ Birmingham B15 2TT, U.K.
}

Chiasma frequencies and distributions were compared in males and females, with and without different types of B-chromosomes, from four Spanish natural populations of Eyprepocnemis plorans. Mean cell chiasma frequency and mean autosomal chiasma frequency were substantially and significantly lower in females than in males. Females also showed altered chiasma distributions compared with males, with fewer proximal chiasmata and more interstitial and distal chiasmata. A variety of sex differences in meiotic chiasmata have now been described in the Orthoptera and these are compared and discussed. $B$-chromosomes had no effects on mean chiasma frequency and relatively little general effect on chiasma distribution, although some minor effects of $\boldsymbol{B}$-chromosomes on chiasma distribution were detected in certain populations and classes of bivalents. In some of these cases $B$-chromosomes affected chiasma distribution in females but not in males.

\section{INTRODUCTION}

Observations on chiasma frequency and distribution in female Orthoptera are hindered by the technical problems posed by the relatively low numbers of oocytes produced and the large amounts of yolk present in oocytes at the time of maturation. These problems were overcome by the development of a suitable squash technique for examining first metaphase nuclei (Perry and Jones, 1974). Subsequently this method, or modifications of it, have been used to study oocyte first metaphases in a number of grasshopper species (Jones et al., 1975; Hewitt, 1976; Fletcher and Hewitt, 1980), but the number of different species examined is still relatively low.

The grasshopper Eyprepocnemis plorans has been intensively studied in recent years, primarily in relation to the $B$-chromosome polymorphism it displays (e.g., Camacho et al., 1980; Henriques-Gil et al., 1982a; Henriques-Gil et al., 1984). There is an obvious interest in extending this study to include oocyte meiosis. An account of the oocyte squash technique as modified for E. plorans and a description of the meiotic sequence and its timing during oocyte maturation have already appeared (Henriques-Gil et al., 1986). The present paper presents a comparative study of chiasma frequency and distribution at first metaphase in males and females from four Spanish natural populations containing different types of $\boldsymbol{B}$-chromosomes.

\section{MATERIALS AND METHODS}

Mature adults of E. plorans were collected from four Spanish natural populations during September 1984. Males were dissected and their testes fixed in acetic alcohol prior to making orcein stained squash preparations (fig. 1(a)). Females were maintained in cages in laboratory conditions until required. Squash preparations of oocyte first metaphases were made using the method of Perry and Jones (1974) as modified by Henriques-Gil et al., (1986) (figs. 1(b)-(d)). Chiasmata were scored from 10 metaphase-I cells from both males and females.

\section{RESULTS}

\section{B-chromosome types}

The main types of $B$-chromosomes, following the nomenclature of Henriques-Gil et al. (1984), 

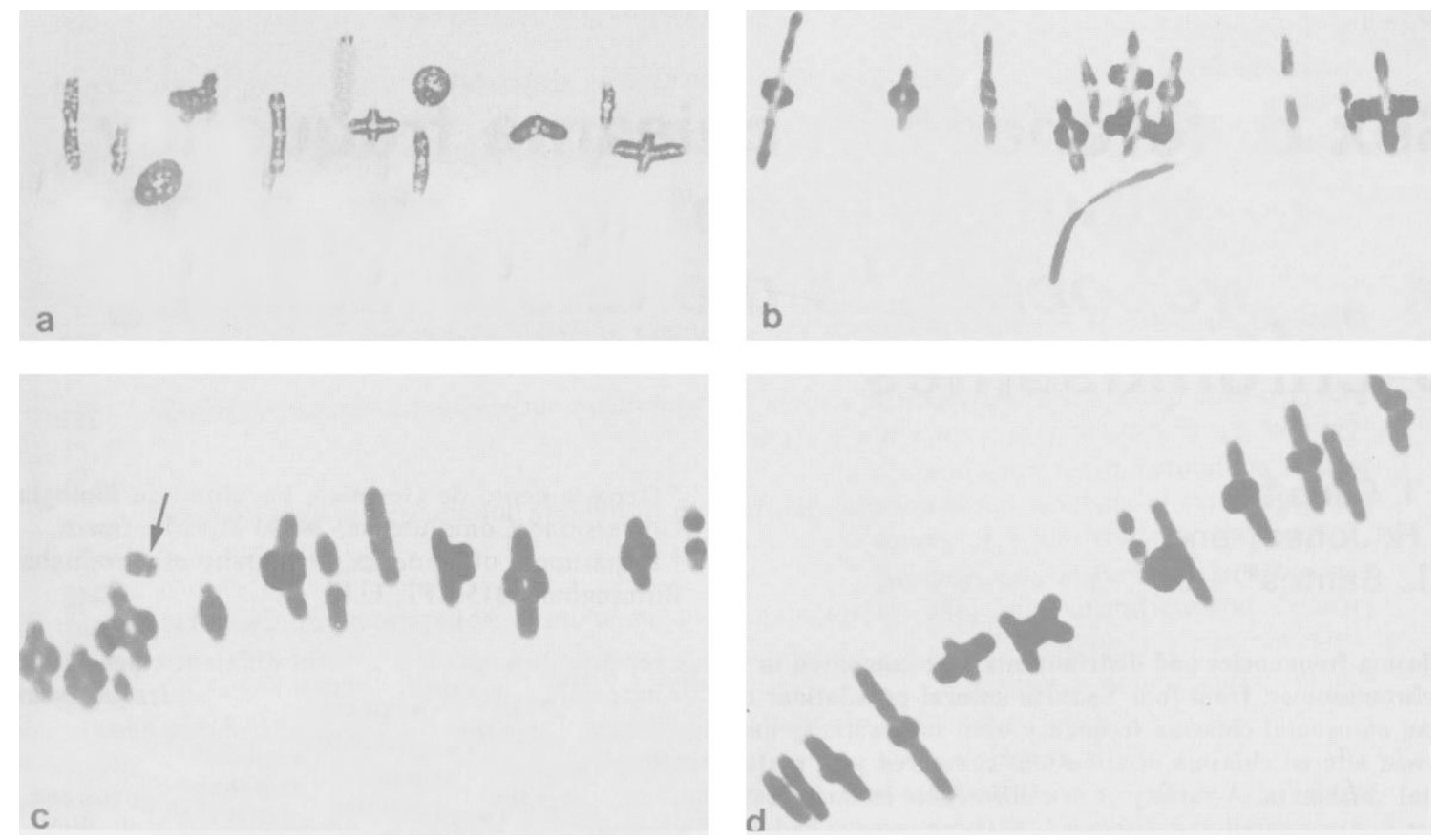

Figure 1 Metaphase $I$ of meiosis in a spermatocyte (a) and oocytes (b)-(d) of E. plorans from population DA. The spermatocyte has eleven bivalents and a univalent $X$-chromosome and shows numerous proximal chiasmata. The oocytes shown in (b)-(d) have 12,13 and 14 chiasmata respectively, which are mainly interstitial to distal, among twelve bivalents. Part (c) shows a single univalent $B$-chromosome (arrowed).

present in the natural populations samples were as follows.

$\begin{array}{ll}\text { Daimuz (Valencia) } & \text { DA type B1 } \\ \text { Torrenueva (Malaga) } & \text { TN type B2 } \\ \text { Malaga (Malaga) } & \text { MA type B1 } \\ \text { Fuengirola } & \text { FG type B5 }\end{array}$

Although one $B$-type predominated in each of these populations, other $B$-types were also present and likely to be included in the samples subjected to meiotic analysis. The low frequencies of some $B$-types, combined with the necessity for Cbanding to distinguish some of them meant that for practical reasons the analyses were restricted to investigating the effects of $B$-chromosomes in general, irrespective of $B$-types.

\section{Mean chiasma frequencies}

Table 1 gives the mean cell chiasma frequencies of male and female grasshoppers having different numbers of $B$-chromosomes, $(0 B, 1 B, 2 B)$ from the four natural populations sampled. These means are based on variable numbers of insects $(n)$, which are also given in table 1 . It should be noted that the female means are based on 12 bivalents, including the $\mathrm{X}$ chromosome pair, while the male means are based on only the 11 autosomal bivalents, the single male $X$-chromosome forming a univalent in spermatocytes. The chiasmata, if any, formed by $B$-chromosomes are not included in these mean chiasma frequencies.

It is apparent from table 1 that the female mean cell chiasma frequencies are generally lower than the corresponding male values; indeed the female population means are lower than the corresponding male population means in every case. Averaging over all $B$ classes and populations, the overall mean cell chiasma frequencies of males and females are $14 \cdot 142$ and $13 \cdot 156$, respectively.

An analysis of variance was performed on the mean chiasma frequencies given in table 1 . No male grasshoppers containing two $B$-chromosomes were sampled in populations $T N$ or $M A$ and therefore, for the purposes of this analysis, it was necessary to decide whether to omit $2 B$ insects from the analysis or to group $1 B$ and $2 B$ insects together into a single " $B$-chromosome" class. The analysis of variance presented in table 2 is based on grouped $B$-chromosome means (i.e., $O B$ vs. $B$ 's) since this involves no loss of data and, in addition, gave identical conclusions to the same analysis performed on the data omitting $2 B$ insects. This analysis (table 2) confirms that there is a large and 
Table 1 Mean chiasma frequencies $(\bar{x})$ and the numbers of insects sampled $(n)$ for males and females of $E$. plorans with different numbers of $B$ chromosomes

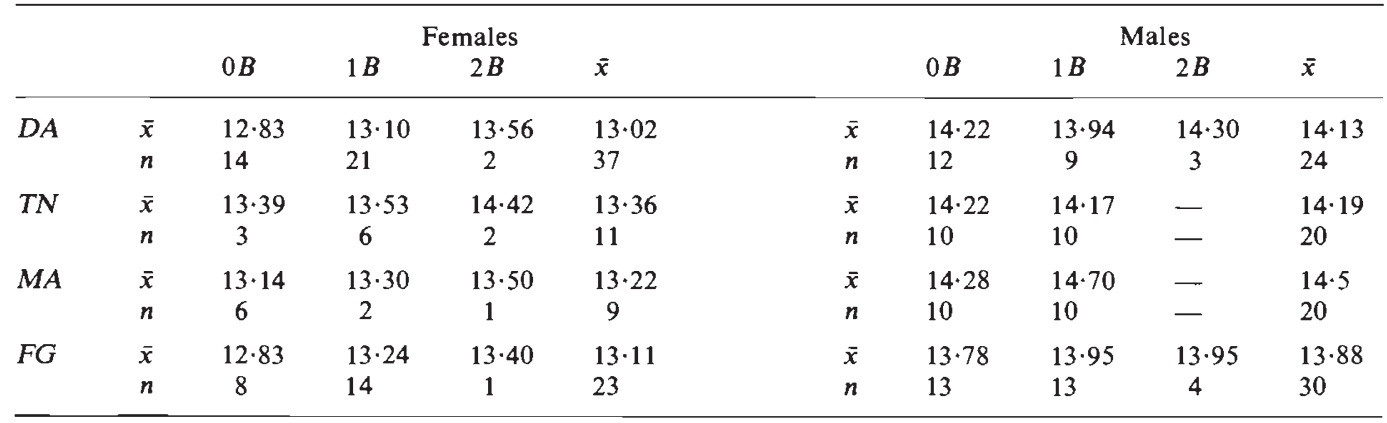

highly significant difference in mean chiasma frequencies between males and females of Eyprepocnemis plorans. In addition the analysis shows that there is no significant effect of $B$ chromosomes on mean chiasma frequency, but there is a just significant $(P<0.05)$ difference in mean chiasma frequency between populations. None of the first order interaction items (sexes $x$ populations; sexes $\times B$-chromosomes; populations $\times B$-chromosomes) are significant and they have therefore been combined with the second order interaction (sexes $\times$ populations $\times B$ chromosomes) to provide a joint error item.

\section{Chiasma distribution}

In order to analyse variation in chiasma distribution within bivalents, all chiasmata scored were assigned by eye to one of 3 regions (proximal, interstitial or distal), each of which occupied one third of each telocentric bivalent. The chiasmata in each of these regions were then summed over insects within $B$ classes $(0 B, 1 B, 2 B)$ and populations. As in the previous analysis of means, $1 B$ and $2 B$ totals were grouped together since no $2 B$ male insects were sampled in two of the four populations. Because it is known from many previous studies, involving a variety of species, that chiasma positions in bivalents are dependent on chiasma number (e.g., Southern, 1967; Shaw and Knowles, 1976) bivalents having one chiasma were separated from those having two chiasmata $(t c)$. In addition, it was possible to separate the one-chiasma bivalents into two sub-classes, the distinct small $(s)$ group, comprising 3 bivalents in each cell, which invariably form one chiasma in both males and females, and the remaining long and medium sized bivalents, including the $X$-bivalent $(\operatorname{lm} x)$. It was not possible to identify the $X$-bivalent in oocyte first metaphases as it was indistinguishable from similar sized autosomal bivalents (Henriques-Gil et al., 1986). The data are presented in table 3 . The chiasma position data were analysed using the log-likelihood ratio statistic (Kullback, 1959). This statistic closely follows the theoretical $\chi^{2}$-distribution and has been used to examine homogeneity of chiasma position in relation to population, sex and presence of $B$-chromosomes. Essentially this procedure tests whether the distribution of chiasmata among proximal, interstitial and distal segments is significantly associated with sex, $B$-chromosome or population differences, either singly or in various combinations. These analyses assume that chiasma distribution is relatively homogeneous between insects in the lowest level groupings (within $B$-classes, within sexes,

Table 2 Analysis of variance of the mean chiasma frequencies of Eyprepocnemis plorans males and females, with and without $B$-chromosomes, from four natural populations

\begin{tabular}{lllllll}
\hline Items & $D F$ & $S S$ & $M S$ & $V R$ & $P$ \\
\cline { 2 - 6 } Sexes & 1 & & 3.658 & 3.658 & 88.571 & $<0.001$ \\
$B$-classes & 1 & 0.175 & 0.175 & 4.237 & NS \\
Populations & 3 & 0.580 & 0.193 & 4.673 & $<0.05$ \\
Sexes $\times$ populations & 3 & 0.238 & 0.079 & & \\
Sexes $\times B$-classes & 1 & 0.058 & 0.058 & & \\
Populations $\times B$-classes & 3 & 0.046 & 0.015 & & \\
Sexes $\times B$-classes $\times$ populations & 3 & 0.071 & 0.024 & & \\
\hline
\end{tabular}


Table 3 The distribution of chiasmata among proximal, interstitial and distal bivalent segments for males and females, with and without $B$ chromosomes in four populations. The data are given separately for $\operatorname{lm} x, s$ and $t c$ bivalents (see text for explanation)

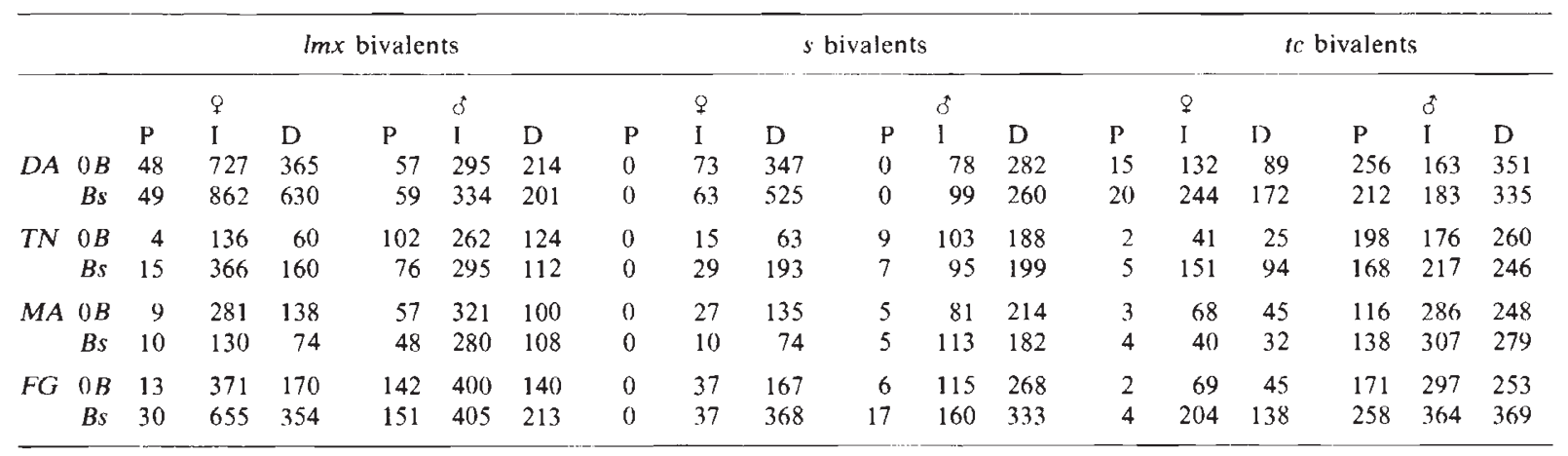

within populations) and this has been shown to be the case. The results of these analyses are summarised in tables 4 and 5 for the 3 bivalent classes separately (i.e., $s, t c$ and $\operatorname{lm} x$ ). As a preliminary step, the higher order associations (chiasma position $\times \operatorname{sex} \times B$ chromosomes $\times$ populations) were examined, since this determines the form of the subsequent analyses. In bivalent classes $s$ and $t c$ this association is non-significant, which simplifies the analyses for these classes of bivalents (table 4) allowing them to be broken down in a straightforward manner to examine the lower order associations. However, in the $\operatorname{lm} x$ bivalent class the higher order association of sex, $B$-chromosomes, populations and chiasma position is highly significant. Further investigation revealed that this is due to an effect of population $D A$, since the higher order association is non-significant when population $D A$ is omitted. To accommodate this, the subsequent analysis of $\operatorname{Im} x$ bivalents was confined to investigating the associations of sex, $B$-chromosomes and chiasma position within each of the four populations (table 5). The results of all the analyses of chiasma positions in the three bivalent classes $(\operatorname{lm} x, s, t c)$ are summarised below.

\section{Effects of sex on chiasma position}

The chiasma position $\times$ sex item is highly significant in all three analyses (see tables 4 and 5). Clearly there is a very marked and consistent effect of sex on chiasma position in all three bivalent classes and in all four populations. Examination of table 3 reveals the nature and extent of this effect. In general, females show proportionally fewer proximal chiasmata and many more interstitial and distal chiasmata in comparison with males, although the chiasma distribution patterns present both in males and females vary according to the bivalent class.

An examination of the second order associations in tables 4 and 5 shows that $B$-chromosomes and population differences can influence the effect of sex on chiasma distribution but these effects are inconsistent over bivalent classes and populations. For example $B$-chromosomes significantly affect the sex difference in chiasma distribution only in the $s$ bivalent class and in population $D A$ for the $\operatorname{lm} x$ bivalents. In both these cases, the presence of $B$-chromosomes seems to enhance the sex difference in chiasma distribution. Population differences significantly influence the effect of sex on chiasma position only in the tc bivalent class.

Table 4 Log-likelihood-ratio tests of association between chiasma position and sex, $B$-chromosomes and populations for the $s$ and $t c$ bivalent classes

\begin{tabular}{|c|c|c|c|c|c|}
\hline & $D F$ & $x^{2}$ & $\begin{array}{l}\text { alents } \\
P\end{array}$ & $x^{2}$ & $\begin{array}{l}\text { lents } \\
P\end{array}$ \\
\hline Position $\times$ sex & 2 & $200 \cdot 57$ & $<0 \cdot 001$ & $650 \cdot 61$ & $<0.001$ \\
\hline Position $\times B$-chromosomes & 2 & 0.66 & $0.7193(\mathrm{NS})$ & $2 \cdot 22$ & 0.3293 (NS) \\
\hline Position $\times$ populations & 6 & 36.92 & $<0.001$ & $148 \cdot 90$ & $<0.001$ \\
\hline Position $\times$ sex $\times B$-chromosomes & 2 & $25 \cdot 12$ & $<0.001$ & 0.82 & $0.6627(\mathrm{NS})$ \\
\hline Position $\times$ sex $\times$ populations & 6 & $6 \cdot 80$ & $0.3399(\mathrm{NS})$ & $37 \cdot 28$ & $<0.001$ \\
\hline Position $\times B$-chromosomes $\times$ populations & 6 & 8.97 & 0.1755 (NS) & $14 \cdot 00$ & $<0.05$ \\
\hline Position $\times$ sex $\times B$-chromosomes $\times$ populations & 6 & $2 \cdot 17$ & 0.9030 (NS) & $2 \cdot 92$ & $0.8188(\mathrm{NS})$ \\
\hline
\end{tabular}


Table 5 A summary of probability values corresponding to log-likelihood ratio statistic for homogeneity of chiasma position and sex and $B$-chromosomes for the $\operatorname{lm} x$ bivalent class. Because of the significant higher order associations, these tests were applied within each of the four populations (see text for full explanation)

\begin{tabular}{lllc}
\hline Populations & $\begin{array}{l}\text { Position } \times \text { sex } \\
\times B \text {-chromosomes }\end{array}$ & Position $\times$ sex & $\begin{array}{l}\text { Position } \\
\times B \text {-chromosomes }\end{array}$ \\
\hline$T N$ & 0.3275 (NS) & $<0.001$ & 0.1060 (NS) \\
$M A$ & 0.1859 (NS) & $<0.001$ & $0.3100(\mathrm{NS})$ \\
$F G$ & 0.2656 (NS) & $<0.001$ & $<0.01$ \\
$D A$ & $<0.001$ & $<0.001$ (within $0 B)<0.001$ (within $\$$ ) \\
& & $<0.001$ (within Bs) 0.3353 (NS) (within $\left.\delta^{\circ}\right)$ \\
\hline
\end{tabular}

\section{Effect of B-chromosomes on chiasma position}

In general there is little evidence of a direct effect of $B$-chromosomes on chiasma position in this study. The chiasma position $\times B$-chromosomes associations were non significant in the $s$ and $t c$ bivalent classes and in populations $M A$ and $T N$ for the $\operatorname{lm} x$ bivalent class, indicating that there is no general effect of $B$-chromosomes on chiasma positions in these cases. Significant general effects of $B$-chromosomes on chiasma position were detected only for $\operatorname{lm} x$ bivalents in population $F G$ and in females of population $D A$. A small effect of populations on the chiasma position $\times B$ chromosome association was detected in the $t c$ bivalent class $(P<0.05)$ despite the absence of a significant association between chiasma position and $B$ chromosomes.

\section{Differences in chiasma position between populations}

Population differences in chiasma position can only be analysed in bivalent classes $s$ and $t c$; because of the significant higher order association this cannot be investigated in $\operatorname{Im} x$ bivalents. In both $s$ and $t c$ bivalents highly significant populations $\times$ chiasma position associations are apparent (table 4). From an examination of table 3 this appears to be due to the presence of distinctive chiasma distributions in males and females of population $D A$. $B$-chromosomes are also associated with this population difference in $t c$ bivalents $(P<0.05)$ but not in $s$ bivalents.

\section{DISCUSSION}

The overall mean cell chiasma frequency of $E$. plorans females is close to one chiasma less than that of males. When allowance is made for the presence of an additional $(X)$ bivalent in females, the difference between the sexes is even more pro- nounced. However, as discussed in earlier papers (Jones et al., 1975; Hewitt, 1976; Fletcher and Hewitt, 1980), the allowance to be made for the $X$-bivalent cannot be determined exactly when the $X$-bivalent itself is indistinguishable from similar sized autosomal bivalents. In the case of E. plorans, it is likely to lie in the range between 1 chiasma (the minimum obligate chiasma possessed by each bivalent) and 1.3 chiasmata (an estimate based on the relative length of the $X$-chromosome and the mean chiasma frequency of oocytes). Consequently it is estimated that the eleven autosomal bivalents of this species form between 2.0 and $2 \cdot 3$ fewer chiasmata in females as compared with males. In addition, this study has demonstrated a pronounced difference in the distributions of chiasmata within bivalents in the two sexes, with females having proportionally more interstitial and distal chiasmata and fewer proximal chiasmata.

It has often been stated, following an observation by Haldane (1922), that where linkage differentials exist between the sexes it is the heterogametic sex in which crossing over is lowered in frequency or absent (e.g., Swanson et al., 1981). While this appears to be supported by the almost universal restriction of achiasmate meiosis to the heterogametic sex in a variety of invertebrate animal groups (White, 1973) there does not appear to be any consistency in this respect among eutherian mammals (Dunn and Bennett, 1967) although two of the most widely studied species, mice and humans, do show the predicted lower male recombination frequencies. Haldane's suggestion is also seriously questioned by the recent finding that heterogametic males of the marsupial insectivore Sminthopsis crassicaudata have much higher recombination frequencies than females (Bennet et al., 1986). This is reflected at the cytogenetical level in a dramatic difference in chiasma frequency and distribution in the two sexes in which females have fewer chiasmata which show marked distal localisation. 
Among Orthoptera three species are known in which the males display extreme localisation of chiasmata, and consequently very restricted recombination, while the females have higher numbers of less localised chiasmata. These species are Stethophyma grossum (White, 1973; Perry and Jones, 1974), Parapleurus alliaceus and Chrysochraon dispar (Fletcher and Hewitt, 1980). The present study shows that $E$. plorans is almost the reverse of these cases in that males have high frequencies of relatively unlocalised chiasmata while females have lower frequencies of more localised chiasmata, although the degree of localisation does not approach that found in males of Stethophyma, Parapleurus and Chrysochraon. Chortoicetes terminifera, the Australian plague locust, appears to be almost a parallel case to E. plorans with females showing a lower chiasma frequency than males and more distally localised chiasmata at least in the medium length bivalents (Fletcher \& Hewitt, 1980). Hewitt (1976) also reported a significantly lower autosomal mean chiasma frequency in females of Myrmeleotettix maculatus, but there was no sex difference in the distribution of chiasmata in this case. Finally, Chorthippus brunneus, the common field grasshopper, shows no significant difference in autosomal chiasma frequency between the sexes but females show a tendency to form more distal chiasmata than males although the difference is not great (Jones et al., 1975).

Urodele amphibians belonging to the genus Triturus also show a diversity of meiotic sex differences (Callan and Perry, 1977). In the palmate newt, Triturus helveticus, male meiosis is characterised by extreme distal localisation of chiasmata, while in females more or less the same numbers of chiasmata are located interstitially in all bivalent arms. In contrast, males of all subspecies of the crested newt, $T$. cristatus, show relatively unlocalised chiasmata distributed along the lengths of most bivalent arms. Females of $T$. cristatus, however, show much lower frequencies of strictly localised chiasmata, but in this case the chiasmata are localised proximally. Males are believed to be the heterogametic sex in both $T$. helveticus (Schmid et al., 1979) and T. cristatus (Sims et al., 1984).

$B$-chromosomes occur fairly commonly among Orthopteran species and a number of studies have investigated the effects of $B$-chromosomes on the chiasma frequencies and distributions of $A$-chromosomes (reviewed by Jones, 1975). In $E$. plorans $B$-chromosomes have been shown to influence both the mean chiasma frequency of $A$ chromosomes and the variance of cell chiasma frequencies (Camacho et al, 1980) but the influence of $B$-chromosomes on chiasma frequency in this species has also been shown to be strongly dependent on the background genotype (Henriques-Gil et al., 1982b). Most investigations of $B$-chromosomes and their effects have been conducted on male meiosis and relatively little is known about their effects on female meiosis. Hewitt (1976) found that $B$-chromosomes cause an increase in chiasma frequency in females of Myrmeleotettix maculatus just as they do in males; however, the effect of $B$-chromosomes on chiasma frequency was additive in females whereas in males $2 B$ s had no more effect than $1 B$.

In the present study the presence of $B$-chromosomes had no overall significant effect on chiasma frequency in males or females of $E$. plorans. Presumably the background genotypes of the insects studied rendered them insensitive to the effects of $B$-chromosomes (Henriques-Gil et al., 1982b). However, the analyses of chiasma distribution revealed some minor effects of $B$-chromosomes on chiasma positions although there was no striking overall effect. A general effect of $B$-chromosomes on chiasma distribution was found only for the $\operatorname{lm} x$ bivalent class and only in population $F G$. More subtle effects, in which $B$-chromosomes affected chiasma distribution in females but not in males were found for the $s$ bivalent class generally and for the $\operatorname{lm} x$ bivalent class in population $D A$. In all these cases the $B$-chromosomes caused a shift to more interstitial and distal chiasma distributions, that is they enhanced the normal sex difference in chiasma distribution.

Interpopulation differences in chiasma frequency have been reported from a variety of plant and animal species (surveyed by Whitehouse et al., 1981). In the present study a small but significant $(P<0.05)$ interpopulation difference in chiasma frequency was found. In general, there is much less information on interpopulation differences in chiasma distribution (Whitehouse et al., $1981)$. In E. plorans the analysis of chiasma distribution has revealed a significant difference between populations which was attributed to the presence of distinctive chiasma distributions in males and females from one population, $D A$. This population appears to be unusual in other respects too since it contributed to the significant higher order association of chiasma position, sex, $B$ chromosomes and populations for the $\operatorname{lm} x$ bivalent class and this population also showed a significant association of chiasma position, sex and $B$ chromosomes which was traced to a significant effect of $B$-chromosomes on chiasma position in 
females but not in males. Evidently this population shows a number of interesting features not found in the other populations sampled. This could be related to the geographical origins of the populations sampled since population $D A$ is from eastern Spain while the other three populations are from southern Spain.

Acknowledgements This work was supported by a grant from the British Council and the Spanish Ministry of Education (Anglo-Spanish Joint Research Programme in higher Education). We are especially grateful to Dr A. J. Birley of the Genetics Department, University of Birmingham, for his help with the statistical analysis, and to Dr P. Arana and Dr N. Henriques-Gil for their valuable advice and comments and for their help in collecting the grasshoppers.

\section{REFERENCES}

BENNETT, J. H., HAYMAN, D. L. AND HOPE, R. M. 1986. Novel sex differences in linkage values and meiotic chromosome behaviour in a marsupial. Nature, 323, 59-60.

CALLAN, H. G. AND PERRY, P. E. 1977. Recombination in male and female meiocytes contrasted. Phil. Trans. R. Soc. Lond. B., 277, 227-233.

CAMACHO, J. P. M., CARBALlo, A. R. AND CABRERO, J. 1980. The $B$ chromosome system of the grasshopper Eyprepocnemis plorans subsp. plorans (Chorpentier). Chromosoma, 80, 163-176.

DUNN, L. C. AND BENNET, D. 1967. Sex differences in recombination of linked genes in animals. Genet. Res., 9, 211-220.

FLETCHER, H. L. AND HEWITT, G. M. 1980. A comparison of chiasma frequency and distribution between sexes in three species of grasshoppers. Chromosoma, 77, 129-144.

HALDANE, J. B. S. 1922. Sex ratio and unisexual sterility in hybrid animals. J. Genet., 12, 101-109.

HENRIQUES-GIL, N., SANTOS, J. L. AND GIRALDEZ, R. $1982 a$. $B$-chromosome polymorphism and interchromosomal chiasma interference in Eyprepocnemis plorans (Acrididae: Orthoptera). Chromosoma, 85, 349-359.
HENRIQUES-GIL, N., SANTOS, J. L. AND GIRALDEZ, R. $1982 b$. Genotype-dependent effect of $B$-chromosomes on chiasma frequency in Eyprepocnemis plorans (Acrididae: Orthoptera). Genetica, 59, 223-227.

HENRIQUES-GIL, N., SANTOS, J. L. AND ARANA, P. 1984. Evolution of a complex $B$-chromosome polymorphism in the grasshopper Eyprepocnemis plorans. Chromosoma, 89, 290 293.

HENRIQUES-GIL, N., JONES, G. H., CANO, M. I., ARANA, P. AND SANTOS, J. L. 1986. Female meiosis during oocyte maturation in Eyprepocnemis plorans (Orthoptera: Acrididae). Can. J. Genet. Cytol., 28, 84-87.

HEWITT, G. M. 1976. Meiotic drive for $B$-chromosomes in the primary oocytes of Myrmeleotettix maculatus (Orthoptera: Acrididae). Chromosoma, 56, 381-391.

JONES, G. H., STAMFORD, W. K. AND PERRY, P. E. 1975. Male and female meiosis in grasshoppers II. Chorthippus brunneus. Chromosoma, 51, 381-389.

JONES, R. N. 1975. B-chromosome systems in flowering plants and animal species. Int. Rev. Cytol., 40, 1-100.

KULLBACK, S. 1959. Information theory and statistics. Wiley, New York.

PERRY, P. E. AND JONES, G. H. 1974. Male and female meiosis in grasshoppers I. Stethophyma grossum. Chromosoma, 47, 227-236.

SCHMID, M., OLERT, J. AND KLETT, C. 1979. Chromosome banding in Amphibia. Chromosoma, 71, 29-55.

SHAW, D. D. AND KNOWLES, G. R. 1976. Comparative chiasma analysis using a computerised optical digitiser. Chromosoma, 59, 103-127.

SIMS, S. H., MACGREGOR, H. C., PELLAT, P. S. AND HORNER, H. A. 1984. Chromosome 1 in crested and marbled newts (Triturus). An extraordinary case of heteromorphism and independent chromosome evolution. Chromosoma, 89, 169-185.

SOUTHERN, D. I. 1967. Chiasma distribution in Truxaline grasshoppers. Chromosoma, 22, 164-191.

SWANSON, C. P., MERZ, T. AND YOUNG, W. J. 1981. Cytogenetics. The chromosome in Division, Inheritance and Evolution. 2nd edition. Prentice-Hall Inc., Englewood Cliffs, N.J.

WHITEHOUSE, C., EDGAR, L. A., JONES, G. H. AND PARKER, J. S. 1981. The population cytogenetics of Crepis capillaris I. Chiasma variation. Heredity, 47, 95-103.

WhIte, M. J. D. 1973. Animal Cytology and Evolution. 3rd edition. University Press, Cambridge. 